CALT-68-2210

nucl-th/9901064

\title{
Radiation pions in two-nucleon effective field theory
}

\author{
Thomas Mehen* and Iain W. Stewart ${ }^{\dagger}$ \\ California Institute of Technology, Pasadena, CA, USA 91125
}

\begin{abstract}
For interactions involving two or more nucleons it is useful to divide pions into three classes: potential, radiation, and soft. The momentum threshold for the production of radiation pions is $Q_{r}=\sqrt{M_{N} m_{\pi}}$. We show that radiation pions can be included systematically with a power counting in $Q_{r}$. The leading order radiation pion graphs which contribute to NN scattering are evaluated in the PDS and OS renormalization schemes and are found to give a small contribution. The power counting for soft pion contributions is also discussed.
\end{abstract}

*mehen@theory.caltech.edu

†iain@theory.caltech.edu 
Effective field theory is a useful method for describing two-nucleon systems [1]. Recently, Kaplan, Savage, and Wise (KSW) [2,3] introduced a power counting which accounts for the effect of large scattering lengths. A similar power counting is discussed in Ref. [4]. According to the KSW power counting, the leading order calculation involves only a dimension 6 , fournucleon operator which is treated nonperturbatively. Higher derivative operators and pion exchange are treated perturbatively. In evaluating diagrams with pions, three types of contributions can be identified: potential, radiation, and soft. Since these pion effects differ in size, they each have a different power counting. This distinction arises because there are several scales associated with two nucleon systems. In this respect the theory is similar to NRQCD and NRQED [5].

In NRQCD there are three mass scales associated with non-relativistic systems containing two heavy quarks: the heavy quark mass $M$, momenta $\sim M v$, and kinetic energy $\sim M v^{2}$, where $v$ is the relative velocity. QCD effects at the scale $M$ are integrated out and appear as local operators in NRQCD. The remaining low energy contributions can be divided into potential, radiation (sometimes referred to as ultra-soft), and soft pieces [6 11]. Potential gluons have energy of order $M v^{2}$ and momentum of order $M v$, radiation gluons have energy and momentum of order $M v^{2}$, and soft gluons have energy and momentum of order $M v$. The power counting for radiation gluons requires the use of a multipole expansion at a quark-gluon vertex [7:9]. The $v$ power counting of potential and radiation gluons can be implemented in the effective Lagrangian by introducing separate gluon fields and rescaling the coordinates and fields by powers of $v[6,8]$. In Ref. [10] the separation of scales was achieved on a diagram by diagram basis using a threshold expansion. The potential, radiation, and soft regimes were shown to correctly reproduce the low energy behavior of relativistic diagrams in a scalar field theory. In Ref. [11] it was pointed out that these effects may be reproduced by an effective Lagrangian in which separate fields are also introduced for the soft regime. Note that soft contributions come from a larger energy scale than potential and radiation effects. The heavy quark system does not have enough energy to radiate a soft gluon, so they only appear in loops. In Ref. [10] it was shown that soft contributions to scattering do not appear until graphs with two or more gluons are considered.

In the nucleon theory there is another scale because the pions are massive. For the purpose of power counting it is still useful to classify pion contributions as potential, radiation, or soft. For a pion with energy $q_{0}$ and momentum $q$, a potential pion has $q_{0} \sim q^{2} / M$ where $M$ is the nucleon mass, while a radiation or soft pion has $q_{0} \sim q \geq m_{\pi}$. In non-relativistic 
theory, integrals over loop energy are performed via contour integration. Potential pions come from contributions from the residue of a nucleon pole and give the dominant contribution to pion exchange between two nucleons. For these pions, the energy dependent part of the pion propagator is treated as a perturbation because the loop energy, $q_{0} \sim q^{2} / M \ll q$. The residues of pion propagator poles give radiation or soft pion contributions. The power counting for soft and radiation pions differs. For instance, the coupling of radiation pions to nucleons involves a spatial multipole expansion, while the coupling to soft pions does not.

In section A, a power counting for graphs with radiation pions is given. To illustrate this power counting, we compute the leading radiation pion contribution to S-wave nucleonnucleon scattering. The power counting for soft pion contributions is discussed in section B, and an illustrative example is given. We emphasize the importance of not double counting when adding radiation and soft pion contributions.

\section{A. Radiation Pions}

In chiral perturbation theory the expansion is in powers of momenta and the pion mass $m_{\pi}$. For power counting potential pions it is convenient to take the nucleon momentum $p=$ $M v \sim m_{\pi}$ [3,6], so $v=m_{\pi} / M \sim 0.15$. The situation is different for radiation pions. There is a new scale associated with the threshold for pion production, which occurs at energy $E=m_{\pi}$ in the center of mass frame. This corresponds to a nucleon momentum $p=Q_{r}$, where $Q_{r} \equiv \sqrt{M m_{\pi}}=360 \mathrm{MeV}$. Because the radiation pion fields cannot appear as on-shell degrees of freedom below the threshold $E=m_{\pi}$, one expects that the radiation pion can be integrated out for $p \ll Q_{r}$. (Potential pions should be included for $p \gtrsim m_{\pi} / 2$.) Another way to see that radiation pions require $p \sim Q_{r}$ is to note that in order to simultaneously satisfy $k_{0}^{2}=k^{2}+m_{\pi}^{2}$ and $k_{0} \sim k \sim M v^{2}$ requires $v \sim \sqrt{m_{\pi} / M} \sim 0.38$.

The full theory with pions has operators in the Lagrangian with powers of $m_{q}$ which give all the $m_{\pi}$ dependence. If the radiation pions are integrated out, then the chiral expansion is no longer manifest because there will be $m_{\pi}$ dependence hidden in the coefficients of operators in the Lagrangian. One is still justified in considering the same Lagrangian, but predictive power is lost since it is no longer clear that chiral symmetry relates operators with a different number of pion fields. Also, the $m_{\pi}$ dependence induced by the radiation pions may affect the power counting of operators. For example, as shown in Ref. [12], integrating out the pion in the one-nucleon sector induces a nucleon electric polarizability $\alpha_{E} \propto 1 / m_{\pi}$. 
Alternatively, one can keep chiral symmetry manifest by working with coefficients in the full theory and including radiation pion graphs. This is the approach we will adopt.

The presence of the scale $Q_{r}$ modifies the power counting of the theory with radiation pions. In the KSW power counting, one begins by taking external momenta $p \sim m_{\pi} \sim Q$. The theory is organized as an expansion in powers of $Q$. To estimate the size of a graph, loop 3-momenta are taken to be of order $Q$. However, potential loops within graphs with radiation pions can actually be dominated by three momenta of order $Q_{r}$. To see how this comes about, consider as an example the graph shown in Fig. 1c. Let $q$ be the momentum running through the pion propagator, and let $k$ be the loop momentum running through a nucleon bubble inside the radiation pion loop. The poles from the pion propagator are

$$
\frac{i}{q_{0}^{2}-\vec{q}^{2}-m_{\pi}^{2}+i \epsilon}=\frac{i}{\left(q_{0}-\sqrt{\vec{q}^{2}+m_{\pi}^{2}}+i \epsilon\right)\left(q_{0}+\sqrt{\vec{q}^{2}+m_{\pi}^{2}}-i \epsilon\right)},
$$

so the radiation pion has $\left|q_{0}\right| \geq m_{\pi}$. This energy also goes into the nucleon bubbles. The $k$ integrand is largest when the nucleons are close to their mass shell. But since the energy going into the loop is $\sim m_{\pi}$, this occurs when $k^{2} / M \sim m_{\pi}$, i.e., $k \sim Q_{r}$. We will begin by considering the contribution of radiation pions to elastic nucleon scattering at the threshold, $E=m_{\pi}$. At this energy, external and potential loop momenta are of the same size and power counting is easiest. Because $p \sim Q_{r}$ it is obvious that we want to count powers of $Q_{r}$ rather than $Q$.

Before discussing the power counting, recall the Lagrangian with pions and nucleons [3]:

$$
\begin{aligned}
\mathcal{L}_{\pi} & =\frac{f^{2}}{8} \operatorname{Tr}\left(\partial^{\mu} \Sigma \partial_{\mu} \Sigma^{\dagger}\right)+\frac{f^{2} \omega}{4} \operatorname{Tr}\left(m_{q} \Sigma+m_{q} \Sigma^{\dagger}\right)+\frac{i g_{A}}{2} N^{\dagger} \sigma_{i}\left(\xi \partial_{i} \xi^{\dagger}-\xi^{\dagger} \partial_{i} \xi\right) N \\
& +N^{\dagger}\left(i D_{0}+\frac{\vec{D}^{2}}{2 M}\right) N-C_{0}^{(s)}\left(N^{T} P_{i}^{(s)} N\right)^{\dagger}\left(N^{T} P_{i}^{(s)} N\right) \\
& +\frac{C_{2}^{(s)}}{8}\left[\left(N^{T} P_{i}^{(s)} N\right)^{\dagger}\left(N^{T} P_{i}^{(s)} \stackrel{\leftrightarrow}{\nabla}{ }^{2} N\right)+\text { h.c. }\right]-D_{2}^{(s)} \omega \operatorname{Tr}\left(m^{\xi}\right)\left(N^{T} P_{i}^{(s)} N\right)^{\dagger}\left(N^{T} P_{i}^{(s)} N\right)+\ldots
\end{aligned}
$$

Here $g_{A}=1.25$ is the nucleon axial-vector coupling, $\Sigma=\xi^{2}$ is the exponential of pion fields, $f=131 \mathrm{MeV}$ is the pion decay constant, $m^{\xi}=\frac{1}{2}\left(\xi m_{q} \xi+\xi^{\dagger} m_{q} \xi^{\dagger}\right)$, where $m_{q}=\operatorname{diag}\left(m_{u}, m_{d}\right)$ is the quark mass matrix, and $m_{\pi}^{2}=w\left(m_{u}+m_{d}\right)$. The matrices $P_{i}^{(s)}$ project onto states of definite spin and isospin, and the superscript $s$ denotes the partial wave amplitude mediated by the operator. This paper will be concerned only with S-wave scattering, so $s={ }^{1} S_{0},{ }^{3} S_{1}$.

The $C_{0}$ operator mediates $\mathrm{S}$-wave nucleon transitions. The $D_{2}$ operator is important because it will be necessary to introduce counterterms proportional to $m_{\pi}^{2}$ to regulate UV 
divergences appearing in the graphs evaluated below. The parameters appearing in Eq. (2) are bare parameters which require renormalization. For systems with two or more nucleons, it is necessary to introduce finite subtractions in order to obtain manifest power counting. Two such renormalization schemes are Power Divergence Subtraction (PDS) [2, [3] and a momentum subtraction scheme (OS) [13]. These renormalization schemes are discussed extensively in Ref. [14]. The renormalized coefficient $C_{0}\left(\mu_{R}\right) \sim 4 \pi /\left(M \mu_{R}\right)$ where $\mu_{R}$ is the renormalization point. A loop with two nucleon propagators gives $\sim M p /(4 \pi)$, so for $\mu_{R} \sim p$ the $C_{0}\left(\mu_{R}\right)$ bubble graphs should be summed. For a scattering length $a$, this summation includes powers of $(a p)$ to all orders [2], as desired since $a$ is large. The coefficients $C_{2}\left(\mu_{R}\right)$ and $D_{2}\left(\mu_{R}\right)$ scale as $\sim 1 / \mu_{R}^{2}$, so for $\mu_{R} \sim p \sim m_{\pi}$ they are treated perturbatively. The ellipsis in Eq. (2) denotes higher order terms including contact interactions which for NN scattering begin to contribute at next-to-next-to-leading order (NNLO). These will not be considered here.

Next, we introduce the power counting at the scale $Q_{r}$. A scheme with manifest power counting will be used, so that $C_{0}\left(\mu_{R}\right) \sim 1 /\left(M \mu_{R}\right), C_{2}\left(\mu_{R}\right) \sim 1 /\left(M \Lambda \mu_{R}^{2}\right)$, etc., where $\Lambda$ is the range of the theory. We will take $p \sim \mu_{R} \sim Q_{r}$. A radiation loop has $q_{0} \sim q \sim m_{\pi}$ so $d^{4} q \sim Q_{r}^{8} / M^{4}$, where $q$ is the momentum running through the pion propagator. A radiation pion propagator gives a $M^{2} / Q_{r}^{4}$, while the derivative associated with a pion-nucleon vertex gives $Q_{r}^{2} / M$. A nucleon propagator gives a $M / Q_{r}^{2}$. External energies and momenta are kept in the nucleon propagator since $E \sim p^{2} / M \sim Q_{r}^{2} / M$. Furthermore, it is appropriate to use a multipole expansion for radiation pion-nucleon vertices which is similar to the treatment of radiation gluons in NRQCD [7]. Therefore, radiation pions will not transfer three-momenta to a nucleon. This is usually equivalent to expanding in powers of a loop momentum divided by $M$ before doing the loop integral. A potential loop will typically have running through it either an external or radiation loop energy $\sim Q_{r}^{2} / M$. Therefore, in these loops the loop energy $k_{0} \sim Q_{r}^{2} / M$, while the loop three momentum $k \sim Q_{r}$, so $d^{4} k \sim Q_{r}^{5} / M$. It is not inconsistent for $k \sim Q_{r}$ while $q \sim Q_{r}^{2} / M$, since three momenta are not conserved at the nucleon-radiation pion vertices. At the scale $Q_{r}$ potential pion propagators may still be treated in the same way, $i /\left(k_{0}^{2}-k^{2}-m_{\pi}^{2}\right)=-i /\left(k^{2}+m_{\pi}^{2}\right)+\mathcal{O}\left(k_{0}^{2} / k^{4}\right)$, which has an expansion in $Q_{r}^{2} / M^{2}$. We will see through explicit examples that this power counting correctly estimates the size of radiation pion graphs.

Note that only the potential loop measure gives an odd power of $Q_{r}$, so without potential loops the power counting reduces to power counting in powers of $m_{\pi}$. The power counting 
a)

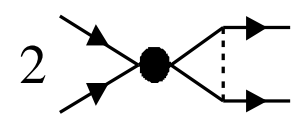

c)

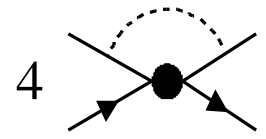

f)

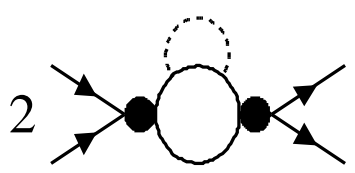

b)

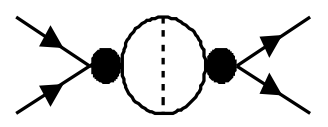

d)
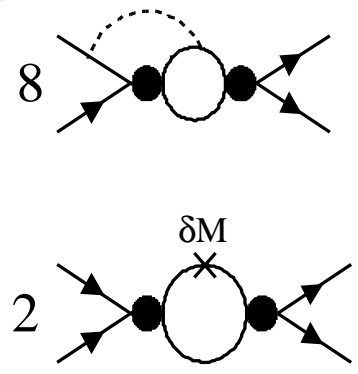

e)
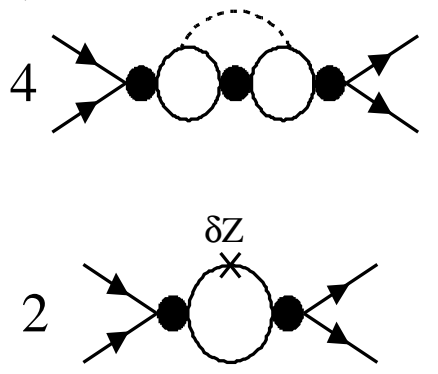

FIG. 1. Leading order radiation pion graphs for $N N$ scattering. The solid lines are nucleons, the dashed lines are pions and $\delta M, \delta Z$ are the mass and field renormalization counterterms. The filled dot denotes the $C_{0}\left(\mu_{R}\right)$ bubble chain. There is a further field renormalization contribution that is calculated in text, but not shown.

here therefore correctly reproduces the usual chiral power counting used in the one nucleon sector 15.

Graphs with one radiation pion and additional higher order contact interactions or potential pions are suppressed by factors of $Q_{r} / \Lambda$ relative to graphs with a single radiation pion and $C_{0}$ vertices. The $Q_{r}$ expansion is a chiral expansion about $m_{\pi}=0$, so there is a limit of QCD where it is justified. The scale $\Lambda$ is unknown. One possible estimate is $\Lambda_{\mathrm{NN}}=8 \pi f^{2} /\left(M g_{A}^{2}\right)=300 \mathrm{MeV}$ since a graph with $m+1$ potential pions is suppressed by $p / 300 \mathrm{MeV}$ relative to a graph with $m$ potential pions. However, this order of magnitude estimate only takes into consideration a partial subset of the graphs of the theory. As argued in Ref. [14], it is possible that the range is of order the scale of short range interactions that are integrated out, implying $\Lambda \sim 500 \mathrm{MeV}$. In fact, the accuracy of NLO computations of nucleon-nucleon phase shifts is in agreement with this physically motivated estimate of the range. We will assume that an expansion in $Q_{r} / \Lambda$ is valid. This hypothesis will be tested further by seeing how well the effective theory makes predictions at $p \sim 300 \mathrm{MeV}$. For example, processes with external pions could be considered. If the $Q_{r} / \Lambda$ expansion is not convergent, then application of the theory is restricted to $p<Q_{r}$.

The radiation pion graphs that give the leading order contribution to nucleon-nucleon scattering are shown in Fig. 1. The filled dot denotes the leading order interaction between nucleons, a $C_{0}\left(\mu_{R}\right)$ bubble sum. We illustrate the power counting with an example, the graph 
in Fig. 1d. For the moment, replace the $C_{0}$ bubble sums with single $C_{0}$ vertices. Each $C_{0}$ gives a factor of $1 / M Q_{r}$ and each nucleon line gives a factor $M / Q_{r}^{2}$. The derivatives from the pion couplings combine with the radiation pion propagator to give a factor of unity. The radiation loop gives $Q_{r}^{8} / M^{4}$, while the nucleon bubble loop gives $Q_{r}^{5} / M$. There is also a factor of $1 / f^{2}$ from pion exchange, and two factors of $1 / 4 \pi$ from the radiation loop giving a $1 / \Lambda_{\chi}^{2}$. ( $\Lambda_{\chi} \sim 1 \mathrm{GeV}$ is the chiral symmetry breaking scale.) Combining all factors, we find that this graph scales like $Q_{r}^{3} /\left(M^{3} \Lambda_{\chi}^{2}\right)$. This graph is suppressed relative to the leading order amplitude, $A^{(-1)}$, by a factor of $Q_{r}^{4} /\left(M^{2} \Lambda_{\chi}^{2}\right)=m_{\pi}^{2} / \Lambda_{\chi}^{2}$. Note that $C_{0}$ bubbles are summed on external nucleon lines as well as in the interior of the radiation loop, and each graph in the sum has the same size. It is straightforward to verify that all graphs in Fig. 1 scale the same way. For external bubble sums we can simply use the vertex $i A^{(-1)}$ where $A^{(-1)}$ is the leading order S-wave amplitude,

$$
A^{(-1)}=-\frac{4 \pi}{M} \frac{1}{\gamma+i p}
$$

and the pole $\gamma=4 \pi / M C_{0}\left(\mu_{R}\right)+\mu_{R} \sim 1 / a$. Graphs with two radiation pions are suppressed by at least $Q_{r}^{8} /\left(M^{4} \Lambda_{\chi}^{4}\right)=m_{\pi}^{4} / \Lambda_{\chi}^{4}$ and will not be considered.

The first graphs we consider are those in Fig. 1 $\mathrm{a}$,b. These graphs have contributions from potential and radiation pions, and it may not be obvious that a clean separation occurs. Here the energy integrals will be evaluated without any approximations, after which the graphs split into radiation and potential parts. The graph in Fig. 11a gives:

$$
i A^{(-1)} \frac{g_{A}^{2}}{2 f^{2}} \int \frac{d^{D} q}{(2 \pi)^{D}} \frac{i}{\frac{E}{2}+q_{0}-\frac{(\vec{q}-\vec{p})^{2}}{2 M}+i \epsilon} \frac{i}{\frac{E}{2}-q_{0}-\frac{(\vec{q}-\vec{p})^{2}}{2 M}+i \epsilon} \frac{i \vec{q}^{2}}{q_{0}^{2}-\vec{q}^{2}-m_{\pi}^{2}+i \epsilon} .
$$

(Throughout this paper we will include a factor of $(\mu / 2)^{4-D}$ in the loop measures.) Performing the $q_{0}$ integral gives a term from the residue of the nucleon pole and a term from the pion pole,

$$
\begin{aligned}
& -i A^{(-1)} \frac{g_{A}^{2}}{2 f^{2}} \int \frac{d^{n} q}{(2 \pi)^{n}} \frac{M}{(\vec{q}-\vec{p})^{2}-M E} \frac{\vec{q}^{2}}{\vec{q}^{2}+m_{\pi}^{2}-\left[\frac{E}{2}-\frac{(\vec{q}-\vec{p})^{2}}{2 M}\right]^{2}} \\
& -i A^{(-1)} \frac{g_{A}^{2}}{4 f^{2}} \int \frac{d^{n} q}{(2 \pi)^{n}} \frac{\vec{q}^{2}}{\sqrt{\vec{q}^{2}+m_{\pi}^{2}}} \frac{1}{\frac{E}{2}+\sqrt{\vec{q}^{2}+m_{\pi}^{2}}-\frac{(\vec{q}-\vec{p})^{2}}{2 M}} \frac{1}{\frac{E}{2}-\sqrt{\vec{q}^{2}+m_{\pi}^{2}}-\frac{(\vec{q}-\vec{p})^{2}}{2 M}},
\end{aligned}
$$

where $n=D-1$. Eq. (5) is the potential pion contribution. Expanding in $\left[\frac{E}{2}-\frac{(\vec{q}-\vec{p})^{2}}{2 M}\right]^{2}=$ $\left[\frac{2 \vec{q} \cdot \vec{p}-\vec{q}^{2}}{2 M}\right]^{2}$ gives the result in Ref. [2],3]. The subleading terms in this expansion are suppressed 
by凹 $m_{\pi}^{2} / M^{2}$. Eq. (可) is the radiation pion contribution. With $|\vec{q}|<M$, we may take $(\vec{q}-\vec{p})^{2} / M \rightarrow p^{2} / M$ in the last two propagators, which is the same approximation that is made by performing the multipole expansion. Finally, we use the equations of motion to set $E-p^{2} / M=0$. It is important to note that we have not neglected $E$ relative to $|\vec{q}|$. For $n=3-2 \epsilon$, Eq. (6) becomes

$$
a)=i A^{(-1)} \frac{g_{A}^{2}}{4 f^{2}} \int \frac{d^{n} q}{(2 \pi)^{n}} \frac{\vec{q}^{2}}{\left(\vec{q}^{2}+m_{\pi}^{2}\right)^{3 / 2}}=-3 i A^{(-1)} \frac{g_{A}^{2} m_{\pi}^{2}}{(4 \pi f)^{2}}\left[\frac{1}{\epsilon}+\frac{1}{3}-\ln \left(\frac{m_{\pi}^{2}}{\bar{\mu}^{2}}\right)\right],
$$

where $\bar{\mu}^{2}=\mu^{2} \pi e^{-\gamma_{E}}$. Note that this integral is finite in three dimensions $(n=2)$.

The next graph we consider is shown in Fig. 1 $\mathrm{b}$. We have chosen to route loop momenta so that $q$ runs through the pion and $\pm k$ and $\pm(k+q)$ run through the nucleon lines. The momentum $k$ is potential, while $q$ can be potential or radiation. Doing the $k_{0}$ contour integral and combining the two terms gives:

$$
\begin{aligned}
-2\left[A^{(-1)}\right]^{2} \frac{g_{A}^{2}}{2 f^{2}} \int \frac{d^{n} k}{(2 \pi)^{n}} & \int \frac{d^{D} q}{(2 \pi)^{D}} \frac{\vec{q}^{2}}{q_{0}^{2}-\vec{q}^{2}-m_{\pi}^{2}+i \epsilon} \frac{1}{E-\frac{\vec{k}^{2}}{M}+i \epsilon} \frac{1}{E-\frac{(\vec{k}+\vec{q})^{2}}{M}+i \epsilon} \\
& \times \frac{E-\frac{(\vec{k}+\vec{q})^{2}}{2 M}-\frac{\vec{k}^{2}}{2 M}}{\left[E-\frac{(\vec{k}+\vec{q})^{2}}{2 M}-\frac{\vec{k}^{2}}{2 M}-q_{0}+i \epsilon\right]\left[E-\frac{(\vec{k}+\vec{q})^{2}}{2 M}-\frac{\vec{k}^{2}}{2 M}+q_{0}+i \epsilon\right]} .
\end{aligned}
$$

Doing the $q_{0}$ integral gives two terms, but the radiation and potential contributions are still mixed. Combining these gives

$$
i\left[A^{(-1)}\right]^{2} \frac{g_{A}^{2}}{2 f^{2}} \int \frac{d^{n} k d^{n} q}{(2 \pi)^{2 n}} \frac{\vec{q}^{2}}{\sqrt{\vec{q}^{2}+m_{\pi}^{2}}} \frac{1}{E-\frac{\vec{k}^{2}}{M}} \frac{1}{E-\frac{(\vec{k}+\vec{q})^{2}}{M}} \frac{1}{E-\frac{(\vec{k}+\vec{q})^{2}}{2 M}-\frac{\vec{k}^{2}}{2 M}-\sqrt{\vec{q}^{2}+m_{\pi}^{2}}},
$$

which can be split into potential and radiation parts

$$
\begin{aligned}
i\left[A^{(-1)}\right]^{2} \frac{g_{A}^{2}}{2 f^{2}} \int \frac{d^{n} k}{(2 \pi)^{n}} & \int \frac{d^{n} q}{(2 \pi)^{n}} \frac{\vec{q}^{2}}{\sqrt{\vec{q}^{2}+m_{\pi}^{2}}} \frac{1}{E-\frac{\vec{k}^{2}}{M}} \frac{1}{\sqrt{\vec{q}^{2}+m_{\pi}^{2}}-\frac{\left(2 \vec{k} \cdot \vec{q}+\vec{q}^{2}\right)}{2 M}} \\
\times & {\left[\frac{-1}{E-\frac{(\vec{k}+\vec{q})^{2}}{M}}+\frac{1}{E-\frac{\vec{k}^{2}}{M}-\frac{\left(2 \vec{k} \cdot \vec{q}+\vec{q}^{2}\right)}{2 M}-\sqrt{\vec{q}^{2}+m_{\pi}^{2}}}\right] . }
\end{aligned}
$$

The first term in Eq. (10) is the two-loop potential pion graph evaluated in Ref. [3]. The factors of $\left(2 \vec{k} \cdot \vec{q}+\vec{q}^{2}\right) /(2 M)$ appearing in the denominators can be dropped because the

${ }^{1}$ Note that $m_{\pi}^{2} / q^{2} \sim m_{\pi} / M$, but we have kept the $m_{\pi}^{2}$ term in the potential pion propagator in Eq. (5). We could consider expanding in $m_{\pi} / q$ using the asymptotic expansion techniques discussed in section $\mathrm{B}$, but for $p \sim m_{\pi}$ these terms would have to be resummed. Unlike the soft and radiation contributions, there is no issue of double counting for potential pions, so for simplicity we will simply keep the $m_{\pi}^{2}$ in the propagator. 
loop integral is dominated by $k, q \ll M$ and therefore $\left(2 \vec{k} \cdot \vec{q}+\vec{q}^{2}\right) /(2 M) \ll \sqrt{\vec{q}^{2}+m_{\pi}^{2}}$. For the second term, which is the radiation pion contribution, this is equivalent to the multipole expansion. Momenta $k \sim \sqrt{M m_{\pi}}$ and $q \sim m_{\pi}$ dominate the integrals in the second term. In Ref. [10], the potential and radiation parts of the graph in Fig. 1 $1 \mathrm{~b}$ were evaluated in the limit $m_{\pi}=0$, and shown to correctly make up the corresponding part of the fully relativistic calculation. The calculation in Ref. [10 agrees with Eq. (10) for $m_{\pi}=0$. Note that the radiation part would not agree if we assumed $k \sim m_{\pi}$ and used static nucleon propagators in the radiation loop?. For $n=3-2 \epsilon$ the radiation part of Eq. (10) is

$$
\begin{aligned}
b) & =i\left[A^{(-1)}\right]^{2} \frac{g_{A}^{2}}{2 f^{2}} \int \frac{d^{n} k}{(2 \pi)^{n}} \int \frac{d^{n} q}{(2 \pi)^{n}} \frac{\vec{q}^{2}}{\vec{q}^{2}+m_{\pi}^{2}} \frac{1}{E-\frac{\vec{k}^{2}}{M}} \frac{1}{E-\frac{\vec{k}^{2}}{M}-\sqrt{\vec{q}^{2}+m_{\pi}^{2}}} \\
& =\left[A^{(-1)}\right]^{2} \frac{g_{A}^{2} M m_{\pi}^{2}}{(4 \pi f)^{2}}\left\{\frac{3 p}{4 \pi}\left[\frac{1}{\epsilon}+\frac{7}{3}-2 \ln 2-\ln \left(\frac{m_{\pi}^{2}}{\bar{\mu}^{2}}\right)-\ln \left(\frac{-p^{2}}{\bar{\mu}^{2}}\right)\right]+\frac{i \sqrt{M m_{\pi}}}{4 \sqrt{\pi}} I_{1}\left(\frac{E}{m_{\pi}}\right)\right\},
\end{aligned}
$$

where

$$
I_{1}(x)=\frac{3}{2} \frac{\Gamma\left(-\frac{5}{4}\right)}{\Gamma\left(\frac{5}{4}\right)}{ }_{3} F_{2}\left(\left\{-\frac{5}{4},-\frac{1}{4}, \frac{1}{4}\right\},\left\{\frac{1}{2}, \frac{5}{4}\right\}, x^{2}\right)+\frac{x \Gamma\left(\frac{1}{4}\right)}{\Gamma\left(\frac{7}{4}\right)}{ }_{3} F_{2}\left(\left\{-\frac{3}{4}, \frac{1}{4}, \frac{3}{4}\right\},\left\{\frac{3}{2}, \frac{7}{4}\right\}, x^{2}\right) .
$$

For $n=2$ the loop integral in Eq. (11) is finite, except for the function $I_{1}$ which has a $p^{2} /(n-2)$ pole. In PDS this pole would effect the running of $C_{2}\left(\mu_{R}\right)$, but we will see that contributions proportional to $I_{1}$ will cancel in the sum of graphs in Fig. 11. Since $A^{(-1)} \sim 1 /\left(M Q_{r}\right)$ the results in Eqs. ([0, 11) are order $Q_{r}^{3} /\left(M^{3} \Lambda_{\chi}^{2}\right)$ as expected. At one-loop the $1 / \epsilon$ pole in Eq. (7) is cancelled by a counterterm

$$
\delta^{\mathrm{uv}, 1 a} D_{2}=-3 C_{0}^{\mathrm{finite}} \frac{g_{A}^{2}}{(4 \pi f)^{2}}\left[\frac{1}{\epsilon}-\gamma_{E}+\ln (\pi)\right] .
$$

For higher loops the $1 / \epsilon$ poles in Eq. (17), Eq. (11), and $\delta^{\mathrm{uv}, 1 a} D_{2}$ dressed with $C_{0}$ bubbles cancel. Note that the $O(\epsilon)$ piece of the bubbles give a finite contribution,

$$
-C_{0}\left(\mu_{R}\right)\left(\frac{M p}{4 \pi}\right)\left\{1+\epsilon\left[2-2 \ln 2-\ln \left(\frac{-p^{2}-i \epsilon}{\bar{\mu}^{2}}\right)\right]\right\} \text {. }
$$

The result of combining Figs. a, b and $\delta^{\mathrm{uv}, 1 a} D_{2}$ dressed by $C_{0}$ bubbles is:

\footnotetext{
${ }^{2}$ Furthermore, if static nucleon propagators are used one obtains a linear divergence requiring a non-analytic counterterm $\propto m_{\pi}$ 16.

${ }^{3}$ The bare coefficients are written as $C^{\text {bare }}=\delta^{\mathrm{uv}} C+C^{\text {finite }}$. In OS and PDS additional finite subtractions are made so that $C^{\text {finite }}=C\left(\mu_{R}\right)-\sum \delta^{n} C\left(\mu_{R}\right)$, see Ref. [14.
} 
$a)+b)=3 i\left[A^{(-1)}\right]^{2} \frac{g_{A}^{2} m_{\pi}^{2}}{(4 \pi f)^{2}} \frac{M \gamma}{4 \pi}\left[\frac{1}{3}-\ln \left(\frac{m_{\pi}^{2}}{\mu^{2}}\right)\right]+i\left[A^{(-1)}\right]^{2} \frac{g_{A}^{2} M m_{\pi}^{2}}{(4 \pi f)^{2}} \frac{\sqrt{M m_{\pi}}}{4 \sqrt{\pi}} I_{1}\left(\frac{E}{m_{\pi}}\right)$

Next we consider the graphs in Fig. 1 c,d,e. The loop integrals in these graphs vanish if the pion pole is not taken so there is no potential pion contribution. As pointed out in Ref. [3], emission of the radiation pion in these graphs changes the spin/isospin of the nucleon pair. Therefore, if the external nucleons are in a spin-triplet(singlet) state, then the coefficients appearing in the internal bubble sum are $C_{0}^{\left({ }^{1} S_{0}\right)}\left(\mu_{R}\right)\left(C_{0}^{\left({ }^{3} S_{1}\right)}\left(\mu_{R}\right)\right)$. The notation $C_{0}\left(C_{0}^{\prime}\right)$ will be used for vertices outside (inside) the radiation pion loop. We begin with Fig. c. The contribution from the graph with $m$ nucleon bubbles in the internal bubble sum is

$$
\begin{aligned}
4 \frac{g_{A}^{2}}{2 f^{2}} \int & \frac{d^{D} q}{(2 \pi)^{D}} \frac{i}{q_{0}-i \epsilon} \frac{i}{q_{0}-i \epsilon} \frac{-i \vec{q}^{2}}{q_{0}^{2}-\vec{q}^{2}-m_{\pi}^{2}+i \epsilon}\left[-i C_{0}^{\prime}\left(\mu_{R}\right)\right]^{m+1} \\
& \times\left[\int \frac{d^{D} k}{(2 \pi)^{D}} \frac{i}{-k_{0}-q_{0}+\frac{E}{2}-\frac{(\vec{k}-\vec{q})^{2}}{2 M}+i \epsilon} \frac{i}{k_{0}+\frac{E}{2}-\frac{\vec{k}^{2}}{2 M}+i \epsilon}\right]^{m}
\end{aligned}
$$

where we used the multipole expansion and then the equations of motion to eliminate $E$ and $p$ from the first two propagators. All nucleon propagators have a $q_{0}$ pole above the real axis, while the pion propagator has one pole above and one below. Therefore, the $q_{0}$ contour is closed below. The $d^{D} k$ integrals are also easily performed giving

$$
-i \frac{g_{A}^{2} C_{0}^{\prime}\left(\mu_{R}\right)}{f^{2}}\left[\frac{-C_{0}^{\prime}\left(\mu_{R}\right) M \Gamma(1-n / 2)}{(4 \pi)^{n / 2}}\right]^{m} \int \frac{d^{n} q}{(2 \pi)^{n}} \frac{\vec{q}^{2}\left[\left(-p^{2}+M \sqrt{\vec{q}^{2}+m_{\pi}^{2}}\right)^{n / 2-1}-\mu_{R}\right]^{m}}{\left(\vec{q}^{2}+m_{\pi}^{2}\right)^{3 / 2}} .
$$

Note that the size of the loop momenta $k$ in the nucleon bubbles is $\sim \sqrt{M m_{\pi}}$ even for $p<\sqrt{M m_{\pi}}$. The $\mu_{R}$ inside the brackets comes from inclusion of the PDS or OS $\delta^{n} C_{0}\left(\mu_{R}\right)$ counterterm graphs for the internal bubble sum. The integral will be dominated by $\vec{q} \sim m_{\pi}$ so the graph will scale as

$$
\frac{1}{\Lambda_{\chi}^{2}} \frac{m_{\pi}^{2}}{M \mu_{R}}\left(\frac{\sqrt{M m_{\pi}}}{\mu_{R}}\right)^{m}
$$

Since $\mu_{R} \sim \sqrt{M m_{\pi}}$, all graphs in the sum are of order $Q_{r}^{3} /\left(M^{3} \Lambda_{\chi}^{2}\right)$.

For Figs. 1c, d,e the sum over bubbles should be done before the radiation loop integral. The reason is that an arbitrary term in the bubble sum has a much different dependence on the energy flowing through it than the sum itself. This can be seen in the $\vec{q}$ dependence in Eq. (17). If we integrate over $\vec{q}$ then terms in the sum may diverge whereas the 
integral of the complete sum is finite. In fact, for $n=3$, Eq. (17) has divergences of the form $\Gamma(-1-m / 4) \mathrm{F}\left(E^{2} / m_{\pi}^{2}\right)$ and $\Gamma(-1 / 2-m / 4) E \mathrm{~F}\left(E^{2} / m_{\pi}^{2}\right)$ where $\mathrm{F}$ is a hypergeometric function. These divergences are misleading because for momenta $>1 / a$ we know that the correct form of the leading order four point function falls off as $1 / p$. For this reason the summation is performed before introducing counterterms to subtract divergences. (This approach is also taken in the analysis of three body interactions in Ref. [17]). Summing over $m$, Eq. (17) becomes:

$$
\begin{aligned}
c) & =-i \frac{g_{A}^{2}}{f^{2}} \frac{4 \pi}{M} \int \frac{d^{n} q}{(2 \pi)^{n}} \frac{\vec{q}^{2}}{\left(\vec{q}^{2}+m_{\pi}^{2}\right)^{3 / 2}} \frac{1}{\gamma^{\prime}-\left[-p^{2}+M \sqrt{\vec{q}^{2}+m_{\pi}^{2}}\right]^{n / 2-1}} \\
& =\frac{i g_{A}^{2}}{\sqrt{\pi} f^{2}}\left(\frac{m_{\pi}}{M}\right)^{3 / 2} I_{2}\left(\frac{E}{m_{\pi}}\right),
\end{aligned}
$$

where $\gamma^{\prime}=4 \pi / M C_{0}^{\prime}\left(\mu_{R}\right)+\mu_{R} \sim 1 / a$. As expected the graph scales as $Q_{r}^{3}$. In the limit $n \rightarrow 3, I_{2}$ is finite and given by

$$
\begin{aligned}
I_{2}(x)= & \frac{\Gamma\left(-\frac{3}{4}\right)}{\Gamma\left(\frac{3}{4}\right)}{ }_{3} F_{2}\left(\left\{-\frac{3}{4}, \frac{1}{4}, \frac{3}{4}\right\},\left\{\frac{1}{2}, \frac{7}{4}\right\}, x^{2}\right)-\frac{3 x}{2} \frac{\Gamma\left(\frac{3}{4}\right)}{\Gamma\left(\frac{9}{4}\right)}{ }_{3} F_{2}\left(\left\{-\frac{1}{4}, \frac{3}{4}, \frac{5}{4}\right\},\left\{\frac{3}{2}, \frac{9}{4}\right\}, x^{2}\right) \\
& +\mathcal{O}\left(\gamma^{\prime} / \sqrt{M m_{\pi}}\right) .
\end{aligned}
$$

$I_{2}$ is manifestly $\mu_{R}$ independent and is also finite as $n \rightarrow 2$.

Next we consider the graph in Fig. 1 d d. Integrals are done in the same manner as that of Fig. 11c. For $n=3-2 \epsilon$, Fig. 11d is

$$
\begin{aligned}
d) & =-4 i A^{(-1)} \frac{g_{A}^{2}}{2 f^{2}} \frac{\Gamma(n / 2-1)}{(4 \pi)^{n / 2-1}} \int \frac{d^{n} q}{(2 \pi)^{n}} \frac{\vec{q}^{2}}{\left(\vec{q}^{2}+m_{\pi}^{2}\right)^{3 / 2}} \frac{\left(-p^{2}+M \sqrt{\vec{q}^{2}+m_{\pi}^{2}}\right)^{n / 2-1}-\left(-p^{2}\right)^{n / 2-1}}{\gamma^{\prime}-\left(-p^{2}+M \sqrt{\vec{q}^{2}+m_{\pi}^{2}}\right)^{n / 2-1}} \\
& =-12 i A^{(-1)} \frac{g_{A}^{2} m_{\pi}^{2}}{(4 \pi f)^{2}}\left[\frac{1}{\epsilon}+\frac{1}{3}-\ln \left(\frac{m_{\pi}^{2}}{\bar{\mu}^{2}}\right)\right]-4 \frac{\left(p-i \gamma^{\prime}\right)}{\sqrt{\pi}} \frac{M A^{(-1)}}{4 \pi} \frac{g_{A}^{2}}{2 f^{2}}\left(\frac{m_{\pi}}{M}\right)^{3 / 2} I_{2}\left(\frac{E}{m_{\pi}}\right) . \quad \quad(21)
\end{aligned}
$$

Fig. 11d is finite for $n=2$, except for the function $I_{1}$. The $1 / \epsilon$ pole in Eq. (21) is cancelled by a new tree level counterterm $i \delta^{\mathrm{uv}, 1 \mathrm{~d}} D_{2} m_{\pi}^{2}$ where $\delta^{\mathrm{uv}, 1 \mathrm{~d}} D_{2}$ has the same form as Eq. (13) except with a -12 instead of a -3 .

Evaluation of Fig. e is also similar to Fig. 凹c. For $n=3-2 \epsilon$ we find:

$$
\begin{array}{rl}
e)=-2 & i \frac{\left(p-i \gamma^{\prime}\right)^{2}}{\sqrt{\pi}}\left[\frac{M A^{(-1)}}{4 \pi}\right]^{2} \frac{g_{A}^{2}}{2 f^{2}}\left(\frac{m_{\pi}}{M}\right)^{3 / 2} I_{2}\left(\frac{E}{m_{\pi}}\right)+i\left[A^{(-1)}\right]^{2} \frac{g_{A}^{2} M m_{\pi}^{2}}{(4 \pi f)^{2}} \frac{\sqrt{M m_{\pi}}}{2 \sqrt{\pi}} I_{1}\left(\frac{E}{m_{\pi}}\right) \\
+ & 12\left[A^{(-1)}\right]^{2} \frac{M p}{4 \pi} \frac{g_{A}^{2} m_{\pi}^{2}}{(4 \pi f)^{2}}\left[\frac{1}{\epsilon}+\frac{7}{3}-2 \ln 2-\ln \left(\frac{m_{\pi}^{2}}{\bar{\mu}^{2}}\right)-\ln \left(\frac{-p^{2}}{\bar{\mu}^{2}}\right)\right] \\
& -6 i\left[A^{(-1)}\right]^{2} \frac{M \gamma^{\prime}}{4 \pi} \frac{g_{A}^{2} m_{\pi}^{2}}{(4 \pi f)^{2}}\left[\frac{1}{\epsilon}+\frac{1}{3}-\ln \left(\frac{m_{\pi}^{2}}{\bar{\mu}^{2}}\right)\right] .
\end{array}
$$


This graph is finite for $n=2$, except for the function $I_{1}$. A $D_{2}$ counterterm cancels the divergence in the last line,

$$
\delta^{\mathrm{uv}, 1 e} D_{2}=6\left(C_{0}^{\mathrm{finite}}\right)^{2} \frac{M \gamma^{\prime}}{4 \pi} \frac{g_{A}^{2}}{(4 \pi f)^{2}}\left[\frac{1}{\epsilon}-\gamma_{E}+\ln (\pi)\right] .
$$

For two and higher loops the remaining $1 / \epsilon$ poles cancel between Eqs. (21,22,23) and $\delta^{\text {uv, }, 1 d} D_{2}$ dressed with $C_{0}$ bubbles, so no new counterterms need to be introduced. The $O(\epsilon)$ piece of the bubbles again give a finite contribution. Combining Figs. П $1 \mathrm{c}, \mathrm{d}, \mathrm{e}$, and $\delta^{\mathrm{uv}, 1 d} D_{2}$ and $\delta^{\mathrm{uv}, 1 e} D_{2}$ dressed with $C_{0}$ bubbles gives

$$
\begin{aligned}
& c)+d)+e)=2 i\left[A^{(-1)}\right]^{2} \frac{g_{A}^{2}}{(4 \pi f)^{2}}\left\{6 m_{\pi}^{2} \frac{M\left(\gamma-\gamma^{\prime} / 2\right)}{4 \pi}\left[\frac{1}{3}-\ln \left(\frac{m_{\pi}^{2}}{\mu^{2}}\right)\right]+\frac{M^{3 / 2} m_{\pi}^{5 / 2}}{4 \sqrt{\pi}} I_{1}\left(\frac{E}{m_{\pi}}\right)\right. \\
& \left.+\frac{\left(\gamma-\gamma^{\prime}\right)^{2}}{2 \sqrt{\pi}} \frac{\left(M m_{\pi}\right)^{3 / 2}}{M} I_{2}\left(\frac{E}{m_{\pi}}\right)\right\}
\end{aligned}
$$

Fig. $1 f$ shows a two loop graph with a nucleon self energy on an internal line. It is important to also include graphs with the one-loop wavefunction and mass renormalization counterterms, $\delta Z, \delta M$ inserted on the internal nucleon line. We will use an on-shell renormalization scheme for defining these counterterms, which ensures that the mass, $M$, appearing in all expressions is the physical nucleon mass. The counterterms are:

$$
\delta M=\frac{3 g_{A}^{2} m_{\pi}^{3}}{16 \pi f^{2}}, \quad \delta Z=\frac{9}{2} \frac{g_{A}^{2} m_{\pi}^{2}}{(4 \pi f)^{2}}\left(\frac{1}{\epsilon}+\frac{1}{3}-\ln \left(\frac{m_{\pi}^{2}}{\bar{\mu}^{2}}\right)\right) .
$$

The result from the graphs in Fig.1ff is then

$$
f)=-3 i\left[A^{(-1)}\right]^{2} \frac{g_{A}^{2}}{(4 \pi f)^{2}} \frac{M^{3 / 2} m_{\pi}^{5 / 2}}{4 \sqrt{\pi}} I_{1}\left(\frac{E}{m_{\pi}}\right) .
$$

When Eq. (26) is added to Eqs. $(15,24)$ the terms proportional to $I_{1}$ cancel. To implement PDS we must consider the value of the graphs in Fig. 1ff using Minimal Subtraction with $n=2$. For $n=2+\epsilon$ we have $\delta M=3 g_{A}^{2} m_{\pi}^{2} \mu /\left(16 \pi f^{2} \epsilon\right)$ and $\delta Z=0$, which makes the sum of graphs in Fig. 1ff finite. Finally, renormalization of the bare nucleon fields in the Lagrangian, $N^{\text {bare }}=\sqrt{Z} N, Z=1+\delta Z$, induces a four-nucleon term

$$
\delta \mathcal{L}=-C_{0}^{(s), \text { finite }}(2 \delta Z)\left(N^{T} P_{i}^{(s)} N\right)^{\dagger}\left(N^{T} P_{i}^{(s)} N\right)
$$

Since $\delta Z \sim Q_{r}^{4} \sim Q^{2}$ this term is treated perturbatively. A tree level counterterm

$$
\delta^{\mathrm{uv}, 0} D_{2}=9 C_{0}^{\mathrm{finite}} \frac{g_{A}^{2}}{(4 \pi f)^{2}}\left[\frac{1}{\epsilon}-\gamma_{E}+\ln (\pi)\right]
$$


is introduced to cancel the $1 / \epsilon$ pole. Dressing the operator in Eq. (27) with $C_{0}$ bubbles gives

$$
-9 i\left[A^{(-1)}\right]^{2} \frac{M \gamma}{4 \pi} \frac{g_{A}^{2} m_{\pi}^{2}}{(4 \pi f)^{2}}\left[\frac{1}{3}+\ln \left(\frac{\mu^{2}}{m_{\pi}^{2}}\right)\right] .
$$

Again, for $n=2$ we have $\delta Z=0$ so no new PDS counterterms were added. Note that if we had instead used bare nucleon fields then there would be no correction of the form in Eq. (27). However, Eq. (26) would be modified because the last graph in Fig. 1ff is no longer present. When this is combined with the contribution from the LSZ formula the sum of Eq. (26) and Eq. (29) is reproduced.

For PDS, the graphs in Figs. П]a-f are finite for $n=2$ so no new finite subtractions were introduced. For $n=3$, counterterms are introduced to renormalize the terms with $\ln \left(\mu^{2}\right)$ in Eqs. (15,24,29) (in PDS $\mu=\mu_{R}$ ). In OS only terms analytic in $m_{\pi}^{2}$ are subtracted [14] (including $\left.m_{\pi}^{2} \ln \left(\mu^{2}\right)\right)$. We find $D_{2}\left(\mu_{R}\right) \rightarrow D_{2}\left(\mu_{R}\right)+\Delta D_{2}\left(\mu_{R}\right)$, with

$$
\Delta D_{2}\left(\mu_{R}\right)=6 C_{0}\left(\mu_{R}\right)^{2} \frac{g_{A}^{2}}{(4 \pi f)^{2}} \frac{M\left(\gamma-\gamma^{\prime}\right)}{4 \pi}\left[-\frac{1}{3}+\kappa+\ln \left(\frac{\mu_{R}^{2}}{\mu_{0}^{2}}\right)\right] .
$$

Here $\kappa=1 / 3$ in PDS and $\kappa=0$ in OS, and $\mu_{0}$ is an unknown scale. Note that the logarithm in Eq. (30) gives a contribution to the beta function for $D_{2}\left(\mu_{R}\right)$ of the form

$$
\beta_{D_{2}}^{(r a d)}=\frac{3 g_{A}^{2}}{4 \pi^{2} f^{2}} \frac{M\left(\gamma-\gamma^{\prime}\right)}{4 \pi} C_{0}\left(\mu_{R}\right)^{2} .
$$

This disagrees with the beta function of Ref. [3], because in that paper the beta function was calculated including only the one-loop graphs.

Adding the contributions in Eqs. (15,24,26,29) gives the total radiation pion contribution to the amplitude at order $Q_{r}^{3}$ :

$$
\begin{aligned}
i A^{r a d}= & 6 i\left[A^{(-1)}\right]^{2} \frac{g_{A}^{2} m_{\pi}^{2}}{(4 \pi f)^{2}} \frac{M\left(\gamma-\gamma^{\prime}\right)}{4 \pi}\left[\kappa+\ln \left(\frac{\mu_{R}^{2}}{m_{\pi}^{2}}\right)\right]-i\left[A^{(-1)}\right]^{2} \frac{\Delta D_{2}\left(\mu_{R}\right) m_{\pi}^{2}}{C_{0}\left(\mu_{R}\right)^{2}} \\
& +i\left[A^{(-1)}\right]^{2}\left[\frac{M\left(\gamma-\gamma^{\prime}\right)}{4 \pi}\right]^{2} \frac{g_{A}^{2}}{\sqrt{\pi} f^{2}}\left(\frac{m_{\pi}}{M}\right)^{3 / 2} I_{2}\left(\frac{E}{m_{\pi}}\right) .
\end{aligned}
$$

The first term here has the same dependence on the external momentum as an insertion of the $D_{2}$ operator dressed by $C_{0}$ bubbles. Its $\mu_{R}$ dependence is cancelled by $\mu_{R}$ dependence in $\Delta D_{2}\left(\mu_{R}\right)$. Note that due to cancellations between graphs, this term is actually suppressed by a factor of $\gamma / Q_{r}$ relative to what one expects from the power counting. The second term in Eq. (32) has a nontrivial dependence on $E$ and is suppressed by an even smaller factor of $\gamma^{2} / Q_{r}^{2}$. These cancellations were not anticipated by the power counting and it would be interesting to determine if the suppression by factors of $\gamma \sim 1 / a$ continues at higher orders. 
a)

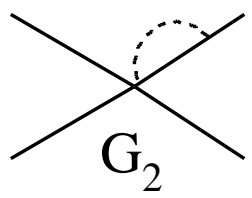

b)

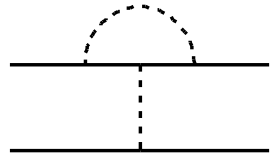

c)

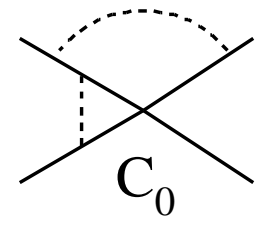

FIG. 2. Examples of order $Q_{r}^{4}$ radiation pion graphs for NN scattering.

If so, this might be a consequence of an additional symmetry of the theory in the limit $a \rightarrow \infty$. If not, terms at order $Q_{r}^{4}$ may actually give the leading contribution of radiation pions to NN scattering.

If we now consider momenta $p \sim m_{\pi} \sim Q \ll Q_{r}$, we should fix $\mu_{R}$ at the threshold, $\mu_{R}=\sqrt{M m_{\pi}}$, and expand in $E / m_{\pi}$ giving $I_{2}\left(E / m_{\pi}\right)=-3.94+\mathcal{O}\left(E / m_{\pi}\right)$. Therefore, the dominant effect of the graphs that occur at order $Q_{r}^{3}$ is indistinguishable from a shift in $D_{2}\left(\mu_{R}\right)$. Integrating out the radiation pions amounts to absorbing their effects into the effective $D_{2}$ in the low energy theory. The result in Eq. (32) is suppressed relative to the NLO contributions in Ref. [3] by a factor of roughly $2\left(\gamma-\gamma^{\prime}\right) / M \sim 1 / 10$. Since this is smaller than the expansion parameter, $Q / \Lambda \sim 1 / 3$, it can be neglected at NNLO.

Recall, that in evaluating the graphs in Fig. 11 a mulitpole expansion is used, which in this case is an expansion in $v=\sqrt{m_{\pi} / M}$. If the first correction in the multipole series were to multiple a nonzero term of order $m_{\pi}^{1 / 2}$ then this would give an order $m_{\pi}$ contribution. However, we have checked that for all the graphs in Fig. 1 1 the first correction in the multipole expansion gives a vanishing contribution.

At order $Q_{r}^{4}$ graphs such as those in Fig. 2 will contribute to NN scattering. The graph in Fig. Za includes an insertion of the operator

$$
\mathcal{L}=i G_{2}\left[N^{T} P_{i}^{(s)} N\right]^{\dagger}\left[N^{T} P_{i}^{(s)} \sigma_{j}\left(\xi \partial_{j} \xi^{\dagger}-\xi^{\dagger} \partial_{j} \xi\right) N\right]+\text { h.c. } .
$$

(Note that due to the hermitian conjugate this operator is the same for $s={ }^{1} S_{0}$ and $s={ }^{3} S_{1}$.) This graph will be dressed with $C_{0}$ bubbles inside and outside the radiation pion loop. The renormalization group equation for $G_{2}$ gives $G_{2}\left(\mu_{R}\right) \sim 1 /\left(M \mu_{R}^{2}\right) \sim 1 /\left(M Q_{r}^{2}\right)$. Combining this with the remaining factors of $Q_{r}$ we find that Fig. 2a is of order $Q_{r}^{4} /\left(M^{4} \Lambda_{\chi}^{2}\right)$ and is therefore suppressed by $Q_{r} / M$ relative to a graph in Fig. 11. Power counting the graphs in Fig. 2]b,c gives $Q_{r}^{4} /\left(M^{3} \Lambda_{\mathrm{NN}} \Lambda_{\chi}^{2}\right)$, giving a factor of $Q_{r} / \Lambda_{\mathrm{NN}}$ relative to a graph in Fig. 11. This provides an example of how graphs with potential pions seem to restrict the range of the effective field theory to $\Lambda_{\mathrm{NN}} \sim 300 \mathrm{MeV}$. The $300 \mathrm{MeV}$ scale applies only to a subset of 
graphs and may change once all graphs at this order are included.

It is important to understand how a $Q_{r}^{m}$ correction scales with $Q$ for $p \sim m_{\pi}$. In Ref. [18] it is demonstrated that $Q_{r}^{m}$ graphs can give a $Q^{m / 2-1}$ contribution plus terms higher order in $Q$. For the $Q_{r}^{3}$ calculations the leading terms scale as $Q^{1 / 2}$ and come from the graphs in Fig. $1 \mathrm{~b}$,d,e. However, it turns out the these $Q^{1 / 2}$ terms cancel. It is not understood at present why this cancellation occurs. Graphs at order $Q_{r}^{4}$ may give an order $Q$ contribution for $p \sim m_{\pi}$ and should be included in a complete NNLO calculation of the NN phase shifts.

\section{B. Soft Pions}

In this section soft pion contributions will be discussed. We will see that there are graphs with non-vanishing soft contributions that should be included for $p \gtrsim m_{\pi}$. In soft loops two scales appear, $m_{\pi}$ and $p=M v$. We will see below that it is necessary to take $p \sim Q_{r}$ when power counting graphs with soft loops in order to avoid double counting. In other words $v$ should have the same value as in the radiation pion calculation. A soft loop has energy and momentum $q_{0} \sim q \sim Q_{r}$, so $d^{4} q \sim Q_{r}^{4}$. The mass of the soft pion is smaller than its momentum, and is treated perturbatively. Nucleon propagators in a soft loop are static (like in heavy quark effective theory, see eg. Ref. [19]) since the loop energy is greater than the nucleon's kinetic energy [11]. Therefore, these propagators count as $1 / Q_{r}$. This power counting is identical to that proposed in Ref. [3] except powers of $Q_{r}$ are counted rather than $Q$.

Unlike potential pions, both soft and radiation pion pieces come from taking the pole in a pion propagator. Therefore, we must be careful not to double count when adding these contributions. This is accomplished by taking $p \sim Q_{r}$ when evaluating both soft and radiation pion graphs. This ensures that the soft and radiation modes have different momenta $\left(\sim Q_{r}\right.$ and $\sim m_{\pi}$ respectively). Integrals involving the scales $Q_{r}$ and $m_{\pi}$ can be separated using the method of asymptotic expansions and dimensional regularization [10,20]. Consider splitting a loop integral into two regimes by introducing a momentum factorization scale $L$ such that $m_{\pi}<L<Q_{r}$. After the pion pole is taken in an energy integral over $q_{0}$, the remaining integral is of the form

$$
\int d^{n} q=\int_{0}^{L} d^{n} q \text { (radiation) }+\int_{L}^{\infty} d^{n} q(\text { soft })
$$

which is obviously independent of $L$. In Eq. (34) the power counting dictates that expansions 
a)

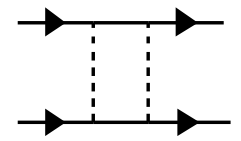

b)

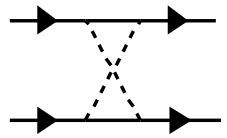

c)

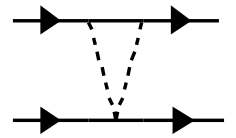

d)

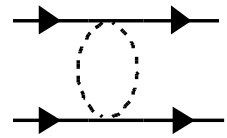

FIG. 3. Examples of one-loop graphs which have soft pion contributions. Graphs a)-d) also have a radiation pion contribution, while in addition graph a) has a potential pion contribution.

in $m_{\pi}^{2} / Q_{r}^{2}$ should be made so that each integral becomes a sum of integrals involving only one scale $\left(m_{\pi}\right.$ for radiation and $Q_{r}$ for soft). In dimensional regularization power divergences vanish, while logarithmic divergences show up as $1 / \epsilon$ poles. Therefore, after expanding we can take $L \rightarrow \infty$ in the radiation integral and $L \rightarrow 0$ in the soft integral. Taking the $L \rightarrow \infty$ and $L \rightarrow 0$ limits may introduce ultraviolet divergences for the radiation integral and infrared divergences for the soft integral. When we add the radiation and soft contributions any superfluous $1 / \epsilon$ poles will cancel. This will be illustrated with an explicit example below. The asymptotic expansion procedure has been rigorously proven for Feynman graphs with large external Euclidean momenta and large masses [21]. It has also been shown to work for the non-relativistic threshold expansion of one and two-loop graphs [10].

Notice that it is crucial to expand the soft pion propagator in powers of $m_{\pi}^{2} / Q_{r}^{2}$, because otherwise the radiation pion contribution may be double counted. As an example, consider the graph in Fig. 1 اa. Taking $p \sim Q_{r}$ implies $M v^{2} \sim m_{\pi}$. For the radiation pion contribution $q_{0} \sim q \sim M v^{2} \sim m_{\pi}$, so we keep the $m_{\pi}^{2}$ in the denominator of Eq. (7). When computing the soft contribution, we assume $q_{0} \sim q \sim Q_{r} \gg m_{\pi}$, and must expand the denominator in powers of $m_{\pi}^{2} / Q_{r}^{2}$. The $\vec{q}$ integration is now scaleless so the soft contribution to Fig. 1a vanishes in dimensional regularization. If we did not expand in $m_{\pi} / Q_{r}$ when evaluating the soft contribution, we would have double counted the radiation contribution. The same argument can be applied to all the diagrams in Fig. 1. In each case the soft contribution vanishes.

Examples of graphs which have a non-vanishing soft contribution are shown in Fig. 3 . These diagrams were calculated in Ref. [22] (although the S-wave channels were not analyzed there). In the KSW power counting they must be dressed on the outside with $C_{0}$ bubbles. (If Fig. 3a or $3 \mathrm{~b}$ are dressed on the inside with $C_{0}$ bubbles then the soft contribution vanishes.) To see how these graphs obtain contributions from the soft and radiation regimes consider Fig. 3a. Unlike the massless case [10], this graph has a radiation contribution. In the ${ }^{1} S_{0}$ 
channel the loop integral for Fig. 3a is

$$
\begin{gathered}
\left(\frac{-i g_{A}^{2}}{2 f^{2}}\right)^{2} \int \frac{d^{D} q}{(2 \pi)^{D}} \frac{i}{\frac{E}{2}+q_{0}-\frac{(\vec{q}+\vec{p})^{2}}{2 M}+i \epsilon} \frac{i}{\frac{E}{2}-q_{0}-\frac{(\vec{q}+\vec{p})^{2}}{2 M}+i \epsilon} \frac{\vec{q}^{2}}{q_{0}^{2}-\vec{q}^{2}-m_{\pi}^{2}+i \epsilon} \\
\times \frac{(\vec{q}-\vec{t})^{2}}{q_{0}^{2}-(\vec{q}-\vec{t})^{2}-m_{\pi}^{2}+i \epsilon},
\end{gathered}
$$

where $\vec{t}=\vec{p}^{\prime}-\vec{p}$ and $\pm \vec{p}$ and $\pm \vec{p}^{\prime}$ are the incoming and outgoing nucleon momenta. Unlike the graphs in Fig. 1, we are forced to route an external momentum, $\vec{t}$, through a pion propagator. Taking a nucleon pole in Eq. (35) gives the potential pion contribution proportional to $M p$. Taking the contribution from the pion poles gives soft and radiation contributions. Our power counting tells us that the leading order soft contribution will be $\sim Q_{r}^{2}$, while the leading order radiation contribution will be $\sim Q_{r}^{4} / M^{2}$. In Eq. (35) the factors of $E / 2-(\vec{q}+\vec{p})^{2} /(2 M)$ can be dropped. In the soft regime the factors of $E / 2-(\vec{q}+\vec{p})^{2} /(2 M)$ are order $Q_{r}^{2} / M$, and are dropped relative to $q_{0} \sim Q_{r}$ leaving static nucleon propagators. In the radiation regime $E / 2-(\vec{q}+\vec{p})^{2} /(2 M) \rightarrow 0$ after using the multipole expansion and equations of motion. This leaves

$$
\begin{aligned}
& \frac{i}{2}\left(\frac{g_{A}^{2}}{2 f^{2}}\right)^{2} \int \frac{d^{n} q}{(2 \pi)^{n}} \frac{\vec{q}^{2}(\vec{q}-\vec{t})^{2}}{\vec{q}^{2}-(\vec{q}-\vec{t})^{2}}\left\{\frac{1}{\left[\vec{q}^{2}+m_{\pi}^{2}\right]^{3 / 2}}-\frac{1}{\left[(\vec{q}-\vec{t})^{2}+m_{\pi}^{2}\right]^{3 / 2}}\right\} \\
& =\frac{i}{2}\left(\frac{g_{A}^{2}}{2 f^{2}}\right)^{2} \int \frac{d^{n} q}{(2 \pi)^{n}} \frac{\vec{q}^{2}(\vec{q}-\vec{t})^{2}}{\left[\vec{q}^{2}+m_{\pi}^{2}\right]^{3 / 2}}\left\{\frac{1}{\vec{q}^{2}-(\vec{q}-\vec{t})^{2}+i \epsilon}+\frac{1}{\vec{q}^{2}-(\vec{q}-\vec{t})^{2}-i \epsilon}\right\},
\end{aligned}
$$

where $n=D-1$. The singularity at $\vec{q}^{2}=(\vec{q}-\vec{t})^{2}$ is cancelled only in the sum of terms in the first line of Eq. (36). These terms can be calculated separately by introducing an $i \epsilon$ in this denominator [10, giving an average over $\pm i \epsilon$ as indicated . The factor of $(\vec{q}-\vec{t})^{2}$ in the numerator can be removed by partial fractioning. For the soft contribution the scale of the loop momentum is set by the external momentum, $q_{0} \sim|\vec{q}| \sim|\vec{t}| \sim Q_{r}$. Expanding Eq. (36) in $m_{\pi}^{2} / \vec{q}^{2}$ gives

$$
\begin{gathered}
i\left(\frac{g_{A}^{2}}{2 f^{2}}\right)^{2} \int \frac{d^{n} q}{(2 \pi)^{n}} \frac{|\vec{q}|}{\left(2 \vec{q} \cdot \vec{t}-\vec{t}^{2} \pm i \epsilon\right)} \sum_{m=0}^{\infty} \frac{\Gamma(-1 / 2)}{\Gamma(-1 / 2-m) \Gamma(m+1)}\left(\frac{m_{\pi}^{2}}{\vec{q}^{2}}\right)^{m} \\
=\frac{-i}{192 \pi^{2}}\left(\frac{g_{A}^{2}}{2 f^{2}}\right)^{2}\left\{\left[\frac{1}{\epsilon}+\ln \left(\frac{\bar{\mu}^{2}}{t^{2}}\right)\right]\left(t^{2}-18 m_{\pi}^{2}\right)-\left[\frac{1}{\epsilon}+\ln \left(\frac{\bar{\mu}^{2}}{t^{2}}\right)\right]\left(90 \frac{m_{\pi}^{4}}{t^{2}}-140 \frac{m_{\pi}^{6}}{t^{4}}+\ldots\right)\right. \\
\left.+\frac{8}{3} t^{2}-36 m_{\pi}^{2}+280 \frac{m_{\pi}^{6}}{t^{4}}+\ldots\right\}
\end{gathered}
$$

\footnotetext{
${ }^{4}$ The second line of Eq. (36) is more easily split into soft and radiation contributions. If we had used the integrand on the first line we would also have to consider $(\vec{q}-\vec{t})^{2} \sim m_{\pi}^{2}$.
} 
where we have kept the first few terms in the expansion. The soft contribution starts at order $Q_{r}^{2}$ as expected. The first $1 / \epsilon$ pole in Eq. (37) is an ultraviolet divergence, while the second is an infrared divergence. For the radiation contribution $q_{0} \sim|\vec{q}| \sim m_{\pi} \ll|\vec{t}|$. Expanding in $(2 \vec{t} \cdot \vec{q}) / \vec{t}^{2}$ gives

$$
\begin{gathered}
-i\left(\frac{g_{A}^{2}}{2 f^{2}}\right)^{2} \int \frac{d^{n} q}{(2 \pi)^{n}} \frac{1}{\left[\vec{q}^{2}+m_{\pi}^{2}\right]^{3 / 2}}\left[\vec{q}^{2}+\frac{\vec{q}^{4}}{\vec{t}^{2}} \sum_{m=0}^{\infty}\left(\frac{2 \vec{q} \cdot \vec{t}}{\vec{t}^{2} \pm i \epsilon}\right)^{m}\right] \\
=\frac{-i}{192 \pi^{2}}\left(\frac{g_{A}^{2}}{2 f^{2}}\right)^{2}\left\{-72 m_{\pi}^{2}\left[\frac{1}{\epsilon}+\ln \left(\frac{\bar{\mu}^{2}}{m_{\pi}^{2}}\right)\right]+\left[\frac{1}{\epsilon}+\ln \left(\frac{\bar{\mu}^{2}}{m_{\pi}^{2}}\right)\right]\left(90 \frac{m_{\pi}^{4}}{t^{2}}-140 \frac{m_{\pi}^{6}}{t^{4}}+\ldots\right)\right. \\
\left.-24 m_{\pi}^{2}+39 \frac{m_{\pi}^{4}}{t^{2}}-\frac{482}{3} \frac{m_{\pi}^{6}}{t^{4}}+\ldots\right\}
\end{gathered}
$$

The radiation contribution starts out as order $Q_{r}^{4} / M^{2}$ as expected. Note that only powers of $m_{\pi}=Q_{r}^{2} / M$ appear. The $1 / \epsilon$ poles in Eq. (38) are ultraviolet divergences. When the soft and radiation contributions are added the infrared poles in Eq. (37) cancel a subset of the ultraviolet poles in Eq. (38). Adding Eq. (37) and Eq. (38) we find

$$
\begin{gathered}
\frac{-i}{192 \pi^{2}}\left(\frac{g_{A}^{2}}{2 f^{2}}\right)^{2}\left\{t^{2}\left[\frac{1}{\epsilon}+\ln \left(\frac{\bar{\mu}^{2}}{t^{2}}\right)\right]+\frac{8}{3} t^{2}-m_{\pi}^{2}\left[\frac{90}{\epsilon}+18 \ln \left(\frac{\bar{\mu}^{2}}{t^{2}}\right)+72 \ln \left(\frac{\bar{\mu}^{2}}{m_{\pi}^{2}}\right)\right]-60 m_{\pi}^{2}\right. \\
\left.+\ln \left(\frac{t^{2}}{m_{\pi}^{2}}\right)\left(90 \frac{m_{\pi}^{4}}{t^{2}}-140 \frac{m_{\pi}^{6}}{t^{4}}+\ldots\right)+39 \frac{m_{\pi}^{4}}{t^{2}}+\frac{358}{3} \frac{m_{\pi}^{6}}{t^{4}}+\ldots\right\},
\end{gathered}
$$

where the remaining ultraviolet $1 / \epsilon$ poles are cancelled by counterterms for $C_{2}$ and $D_{2}$.

If we are interested in making predictions for $p \sim m_{\pi}$, then powers of $m_{\pi}^{2} / t^{2}$ must be summed up. Summing the series in Eq. (39) gives

$$
\begin{aligned}
3 a)=\frac{-i}{192 \pi^{2}}\left(\frac{g_{A}^{2}}{2 f^{2}}\right)^{2}\left\{\left(t^{2}-90 m_{\pi}^{2}\right)\left[\frac{1}{\epsilon}+\ln \left(\frac{\bar{\mu}^{2}}{m_{\pi}^{2}}\right)\right]+\frac{8}{3} t^{2}-58 m_{\pi}^{2}\right. \\
\left.-\frac{\left(128 m_{\pi}^{4}+16 m_{\pi}^{2} t^{2}-t^{4}\right)}{t \sqrt{t^{2}+4 m_{\pi}^{2}}} \ln \left(\frac{\sqrt{t^{2}+4 m_{\pi}^{2}}-t}{\sqrt{t^{2}+4 m_{\pi}^{2}}+t}\right)\right\} .
\end{aligned}
$$

Since the coefficients in the series in Eqs. (37,38) diverge for $\vec{p}^{\prime}=\vec{p}$, Eq. (40) should be used when integrating over $d t$ to obtain the ${ }^{1} S_{0}$ partial wave amplitude (even for $p \sim Q_{r}$ ). Eq. (40) agrees with the result of evaluating Eq. (36) exactly. Although the asymptotic expansion is not necessary for evaluating Fig. 3a, it allows us to identify the radiation and soft contributions to this graph and verify that the power counting for each regime works. We also see that adding soft and radiation pions reproduces the correct answer without double counting. Recall that power counting with $p \sim Q_{r}$ was necessary to avoid double counting for graphs like those in Fig. 1. 
For $p \sim Q_{r}$ the diagrams in Fig. 3 are order $Q_{r}^{2} /\left(f^{2} \Lambda_{\chi}^{2}\right)$, and are larger than the order $Q_{r}^{3}$ graphs with a single radiation pion in Fig. 11. Decreasing $p$ to $p \sim m_{\pi}$ the graphs in Fig. 通 give contributions of the form

$$
\frac{\vec{t}^{2}}{f^{2}(4 \pi f)^{2}} F\left(\vec{t}^{2} / m_{\pi}^{2}\right),
$$

where $F$ is a function. For $p \sim m_{\pi}$ the graphs in Fig. 3 are order $m_{\pi}^{2}$ which is smaller than the graphs in Fig. 10 which include order $m_{\pi}^{1 / 2}, m_{\pi}$, and $m_{\pi}^{3 / 2}$ terms. It is interesting to note that the relative importance of these graphs changes with $p$. The graphs in Fig. 3 dressed by $C_{0}$ bubbles give a contribution that is the same size as a four nucleon operator with 6 derivatives, $C_{6}\left(\mu_{R}\right) p^{6}$, and are $\mathrm{N}^{3} \mathrm{LO}$ in the KSW power counting.

It would be nice to see the expansion used in evaluating the radiation contribution to Fig. 政 implemented at the level of the Lagrangian. It is not clear to us how to do this at the present time. In the radiation regime, the pion whose pole is taken can be thought of as a radiation pion. However, the other propagator gives factors of $1 / t^{2}, \vec{q} \cdot \vec{t} / t^{4}$, etc., which look more like insertions of non-local operators than the propagator of a field. Also, since in general $\vec{p} \neq \vec{p}^{\prime}$, the couplings for this second propagator change the nucleon momenta and therefore do not involve a multipole expansion. Finally, the result in Eq. (40) does not have an expansion in $E / m_{\pi}$. So unlike the radiation pion contribution computed in section A, this contribution cannot be integrated out for $p<\sqrt{M m_{\pi}}$. For these reasons, the use of the term radiation for this contribution differs somewhat from the usage in section A.

To summarize, we have introduced a power counting in factors of $Q_{r}=\sqrt{M m_{\pi}}$ appropriate for graphs with radiation pions. The order $Q_{r}^{3}$ radiation contributions to NN scattering were computed and found to be suppressed by inverse powers of the scattering length. Soft pion contributions also have a power counting in $Q_{r}$. For $p \sim Q_{r}$ they are $\sim Q_{r}^{2}$, but are higher order than the radiation contributions for $p \sim m_{\pi}$. Higher order corrections are suppressed by factors of $Q_{r} / \Lambda$ and whether or not this expansion is convergent is an open question. If the range of the two-nucleon effective field theory with perturbative pions is really $300 \mathrm{MeV}$, and the suppression by factors of $\gamma$ does not persist at higher orders then contributions from radiation pions induce an incalculable error of order $m_{\pi}^{2} / \Lambda_{\chi}^{2}$ to the NN scattering amplitude in this theory. The validity of the $Q_{r} / \Lambda$ expansion can be tested by looking at processes at $p \sim 300 \mathrm{MeV}$ such as those with external pions.

We would like to thank Mike Luke and Mark Wise for several enlightening discussions, as well as S. Fleming, D.B. Kaplan and U. van Kolck for their comments. We also thank H. 
Griesshammer for emphasizing the importance of soft pions. This work was supported in part by the Department of Energy under grant number DE-FG03-92-ER 40701. T.M. was also supported by a John A. McCone Fellowship. 


\section{REFERENCES}

[1] S. Weinberg, Phys. Lett. B251 (1990) 288; Nucl. Phys. B363 (1991) 3; C. Ordonez and U. van Kolck, Phys. Lett. B291 (1992) 459; C. Ordonez, L. Ray and U. van Kolck, Phys. Rev. Lett. 72 (1994) 1982; Phys . Rev. C53 (1996) 2086; U. van Kolck, Phys. Rev. C49 (1994) 2932. G.P. Lepage, nucl-th/9706029. D.B. Kaplan, M.J. Savage, and M.B. Wise, Nucl. Phys. B478 (1996) 629.

[2] D.B. Kaplan, M.J. Savage, and M.B. Wise, Phys. Lett. B424 (1998) 390.

[3] D.B. Kaplan, M.J. Savage, and M.B. Wise, Nucl. Phys. B534 (1998) 329.

[4] U. van Kolck, hep-ph/9711222 and nucl-th/9808007.

[5] W.E. Caswell and G.P. Lepage, Phys. Lett. B167 (1986) 43 7; G.T. Bodwin, E. Braaten, and G.P. Lepage, Phys. Rev. D55 (1997) 1125.

[6] M. Luke and A. Manohar, Phys. Rev. D55 (1997) 4129.

[7] B. Grinstein and I. Rothstein, Phys. Rev. D 57 (1998) 78.

[8] M. Luke and M.J. Savage, Phys. Rev. D57 (1998) 413.

[9] P. Labelle, Phys. Rev. D58 (1998) 093013.

[10] M. Beneke and V.A. Smirnov, Nucl. Phys. B522 (1998) 321.

[11] H.W. Griesshammer, Phys. Rev. D58 (1998) 094027; hep-ph/9810235.

[12] V. Bernard, N. Kaiser, and U. Meissner, Phys. Rev. Lett. 67, 1515 (1991); Nucl. Phys. B 373, 364 (1992); Phys. Lett. B 319, 269 (1993).

[13] J. Gegelia, nucl-th/9802038; T. Mehen, and I. Stewart, Phys. Lett. B445 (1999) 378.

[14] T. Mehen and I. Stewart, nucl-th/9809095.

[15] E. Jenkins and A. Manohar, Phys. Lett. B255 (1991) 558, ibid B 259 (1991) 353.

[16] N. Shoresh and G. Rupak, private communication.

[17] P.F. Bedaque and U. van Kolck, Phys. Lett. B428 (1998) 221; P.F. Bedaque, H.W. Hammer, and U. van Kolck, Phys. Rev. C58 (1998) R641.

[18] T. Mehen and I. W. Stewart, nucl-th/9906010.

[19] M. Neubert, Phys. Rep. 245, 259 (1994).

[20] S.G. Gorishny, Nucl. Phys. B319 (1989) 633; G.B. Pivovarov and F.V. Tkachov, Int. Journ. Mod. Phys. A8 (1993) 2241; V.A. Smirnov, Phys. Lett. B394 (1997) 205; A. Czarnecki and V.A. Smirnov, Phys. Lett. B394 (1997) 211; V.A. Smirnov and E.R. Rakhmetov, hep-ph/9812529.

[21] K.G. Chetyrkin, Teor. Mat. Fiz. 75 (1998) 26; ibid 76 (1998) 207; K.G. Chetyrkin and V.A. Smirnov, Phys. Lett. B144 (1984) 419; V.A. Smirnov, Commun. Math. Phys. 134 (1990) 109; V.A. Smirnov, Renormalization and asymptotic expansions, Birkhäuser, Basel, 1991.

[22] N. Kaiser, R. Brockmann, and W. Weise, Nucl. Phys. A625 (1997) 758. 